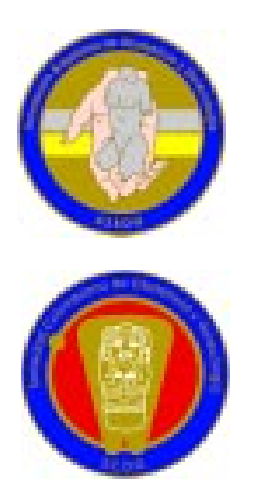

CAso clínico

\title{
DIAGNÓSTICO ECOGRÁFICO PRENATAL DE LA PENTALOGÍA DE CANTRELL
}

\author{
PRENATAL UILTRASONOGRAPHIC \\ DIAGNOSIS OF CANTRELLIS PENTALOGY \\ Hernán Cortés Y., M.D. , José Fernando Vélez V., M.D. ${ }^{* *}$ \\ Recibido: junio 7/2002 - Aceptado: octubre 30/2002
}

\section{RESUMEN}

En 1958, Cantrell describió un síndrome caracterizado por cinco defectos que incluyen: ectopia cordis, onfalocele, disrupción del esternón distal, diafragma anterior y pericardio diafragmático. Se presenta un caso de pentalogía de Cantrell con diagnóstico ecográfico tardío, ecocardiografía luego del nacimiento y estudio post mórtem. Se hace además una corta revisión de la literatura.

Palabras clave: pentalogía de Cantrell, onfalocele, ectopia cordis, ecografía prenatal, ecocardiografía.

\section{SUMMARY}

In 1958, Cantrell described a syndromic complex characterized by five defects, these included: ectopia cordis, omphalocele, disruption of the distal sternum, anterior diaphragm, and diaphragmatic pericardium.

Obstetra y ginecólogo U de A.

* Obstetra y ginecólogo U de A. Ecocardiografista fetal
We present a case of Pentalogy of Cantrell with a late sonographic diagnosis with echocardiography after birth and post-mortem examination. We do a short review of literature.

Key words: Pentalogy of Cantrell, omphalocele, ectopia cordis, prenatal sonography, echocardiography.

\section{INTRODUCCIÓN}

En 1958, el doctor Cantrell, describió un síndrome que lleva su nombre, desde entonces se han reportado varios casos en la literatura, de diagnóstico precoz y con terminación antes de la viabilidad.

Se describe un caso de pentalogía de Cantrell, de diagnóstico ecográfico tardío y con estudios ecocardiogáfico y post mórtem.

\section{REPORTE DE UIN CASO}

Mujer de 19 años, primigestante, con fecha de última menstruación desconocida, a quien remiten de otra institución, por actividad uterina y de edad gestacional desconocida.

Se le realiza ecografía con ecógrafo de tiempo real, con trasductor 3,5 Mhz, encontrando un feto 
único en cefálica, con diámetro biparietal y longitud de fémur para 35 semanas de gestación; el cual presentaba un gran defecto en la pared abdominal, con extrusión de hígado, intestino, estómago y corazón; se observó además un corazón anormal, con cavidades izquierdas hipoplásicas. (Ver figuras 1 a 3). Se hace un diagnóstico de onfalocele gigante y posible pentalogía de Cantrell.

Se envía interconsulta a Cirugía Infantil, quienes recomiendan cesárea electiva y ecocardiografía post-natal al bebé, para confirmar o descartar la hipoplasia cardiaca, para definir el manejo y pronóstico.

Tres días después, por trabajo de parto activo, se realiza cesárea, obteniéndose un bebe de sexo femenino, de $2900 \mathrm{~g}, 44 \mathrm{~cm}$ de talla, con gran defecto en la pared abdominal anterior, cubierto por una membrana, en la que se observan latidos cardiacos, con Apgar de 7 al minuto y de 4 a los cinco minutos, con 37 semanas por Capurro.

El bebé se hospitaliza en la unidad neonatal, donde presenta deterioro progresivo del estado general, requiriendo soporte ventilatorio. Es evaluado por el servicio de Cirugía Infantil, quienes recomiendan manejo de soporte.

Se realiza ecocardiografía que reportó, aurícula común, válvula $\mathrm{A}-\mathrm{V}$ común, doble salida del ventrículo derecho, aorta dilatada, arteria pulmonar de menor tamaño, rama derecha e izquierda de 4 y $5 \mathrm{~mm}$ respectivamente, defecto septal, ventrículo izquierdo hipoplásico, aorta descendente normal y corazón fuera de la cavidad torácica. (Ver figuras 4 y 5).

La bebé fallece al tercer día.

El estudio anatomopatológico reveló un defecto en la pared abdominal tipo onfalocele, en el que se alcanzan a identificar vísceras huecas y macizas; en el examen interno se encontró que el esternón está acortado, faltan las costillas flotantes, el diafragma sólo se identifica en su tercio

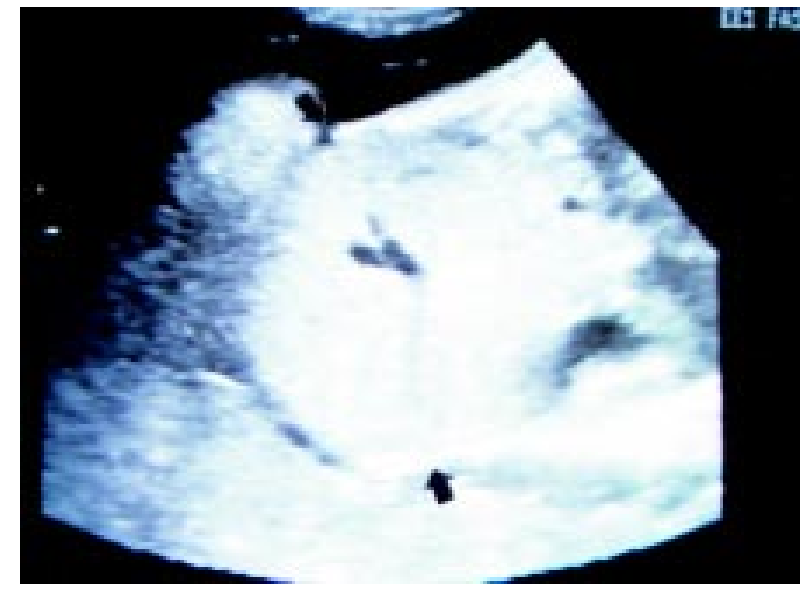

Figura 1. Las flechas (4) señalan los bordes del defecto y el hígado a través de aquel.

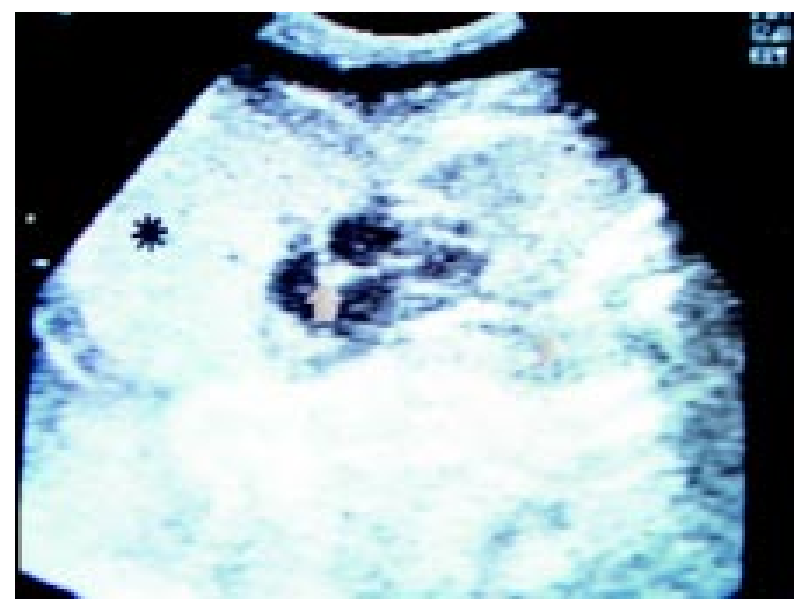

Figura 2. Corte transversal, se observa el hígado (*) y el corazón (†) saliendo del tórax.

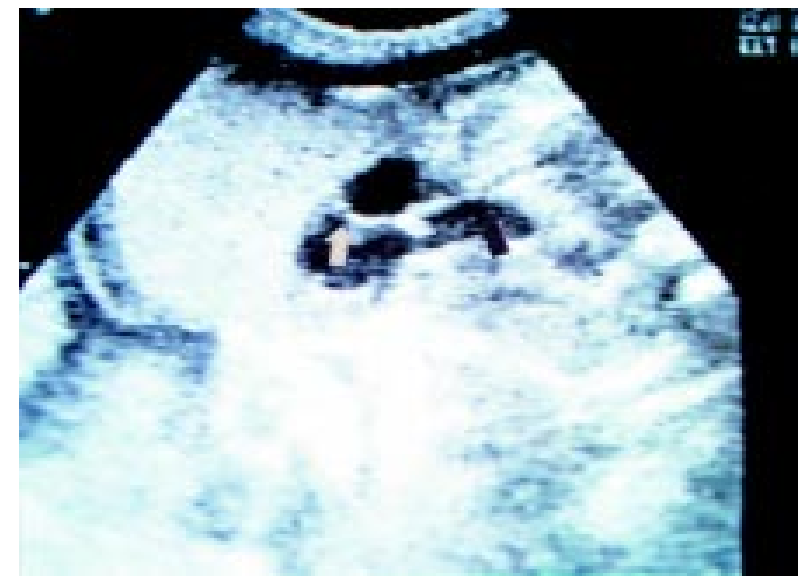

Figura 3. Corazón se observa la arteria pulmonar (4) y la válvula $\mathrm{A}-\mathrm{V}$ del ventrículo derecho (tricúspide) (4). 


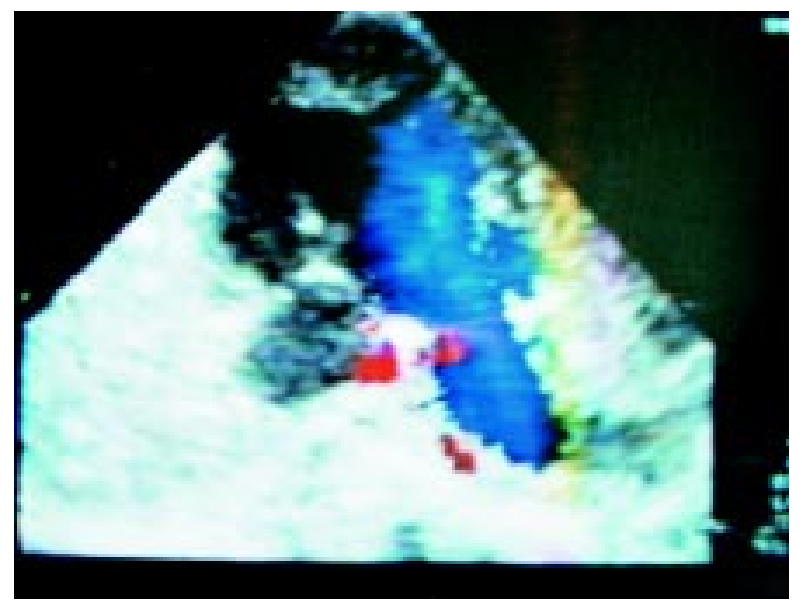

Figura 4. Flujo a través de la arteria pulmonar, ventrículo izquierdo hipoplásico.

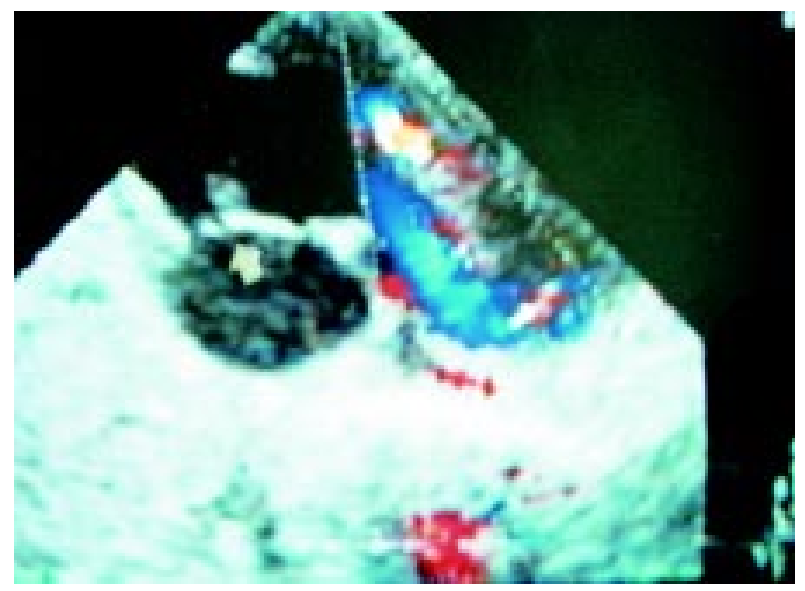

Figura 5. Flujo a través de la arteria pulmonar, se observa la válvula A-V (†).

posterior dejando comunicadas las vísceras torácicas y abdominales, el pulmón derecho es bilobulado con dos bronquios fuente, la punta del corazón sobresale en el defecto de la pared abdominal y tiene una aurícula única, un ventrículo izquierdo hipoplásico una arteria pulmonar que en su origen es anómala y atrésica, con una válvula de solo dos valvas y el hígado que ocupa gran parte del defecto de la pared abdominal, tiene su cara anterior, con una convexidad prominente.

No se encontraron malformaciones en el sistema nervioso central, ni en el tracto gastrointestinal, ni en el aparato urinario.

\section{DISCUSIÓN}

En 1958, Cantrell y cols. describieron una combinación de cinco anomalías congénitas como parte de un complejo síndrome que incluye ectopia cordis, onfalocele, defecto inferior del esternón, defecto del diafragma anterior y defecto en el pericardio diafragmático, asociado con malformaciones cardíacas principalmente defectos en los septos auricular y/o ventricular y tetralogía de Fallot, entre otros. ${ }^{1,2}$

La etiología de esta enfermedad es desconocida; se ha propuesto que este síndrome se debe a una falla en el desarrollo del mesodermo lateral entre los días 14 y 18 de vida embrionaria, lo que produce un fracaso en el cierre de la pared abdominal, del esternón distal, con aplasia del septo que forma el diafragma anterior y el pericardio diafragmático produciéndose la extrusión del corazón y los órganos abdominales a través de este defecto. ${ }^{2,3,4}$

Es importante anotar que el desarrollo embriológico de estos defectos es complejo; por tanto, se han descrito variantes de este síndrome.

Abu-Yousef y cols. reportaron un caso sin defectos en el esternón ni en el pericardio, que se terminó a la semana $21^{\mathrm{a}}$ con múltiples anomalías

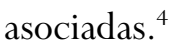

Ghidini y cols. realizaron una revisión de diez casos, dos de los cuales no tenían defectos en el pericardio; además reportaron múltiples anomalías asociadas, como cefalocele, paladar hendido, clinodactilia, cifoescoliosis, hipoplasia pulmonar y cordones umbilicales de dos vasos, entre otras, con defectos cardíacos tipo CIA, CIV, tetralogía de Fallot y estenosis pulmonar, con mortalidad del 100\%, dos casos de trisomía 18 y una proporción mujer hombre de 10:7.

Toyama encontró que un 18\% de estos casos estaban asociados con anomalías faciales y del cráneo. ${ }^{6}$ Además, se ha reportado que este síndrome se asocia con la trisomía 13 y síndrome de Turner. 
En la literatura revisada, no se encontró ningún reporte de pulmón derecho con dos lóbulos ni agenesia de las costillas falsas como en este caso, además esta bebé no tenía anomalías faciales, craneales, ni en las extremidades, lo cual ocurre frecuentemente, pero sí presentaba varios defectos cardíacos severos.

El diagnóstico ecográfico prenatal de esta patología es fácil debido al gran defecto y su rápida aparición, por lo que se debe sospechar en todo onfalocele asociado a extrusión cardiaca y su diagnóstico debe llevar a la búsqueda de anomalías asociadas y a una cuidadosa evaluación de la anatomía cardiaca, pues esto, junto con el estudio cromosómico, define el pronóstico neonatal.

El pronóstico es muy pobre cuando se cumplen todos los criterios incluyendo los defectos cardíacos, por lo que se recomienda la terminación del embarazo en los casos diagnosticados antes de alcanzar la viabilidad fetal. Si el diagnóstico se realiza en el tercer trimestre, se debe favorecer el parto vaginal.

En casos menos severos, se debe realizar un manejo multidisciplinario que incluye al pediatra y al cirujano infantil para definir el momento y el tipo de parto más adecuado y la cirugía para el defecto.
No se han reportado recurrencias de este síndrome en los embarazos subsecuentes.

\section{BIBLIOGRAFÍA}

1. Cantrell JR, Ravitch MM. A syndrome of congenital defects involving the abdominal wall, sternum, diaphragm, pericardium and heart. Surg Gynecol Obstet. 107: 602, 58.

2. Benacerraf, B., Prenatal diagnosis of fetal syndromes. Churchill Livingstone, 1998; pp. 267-269.

3. Moore, KL. The developing human: Clinically oriented embryology. $4^{\text {th }}$ edition. Philadelphia: Saunders; 1988.

4. Abu-Yousef, M., Wray, A., Williamson, R., Bonsib, S., Antenatal ultrasound diagnosis of variant of Pentalogy of Cantrell. J Ultrasound Med 1987; 6:535538.

5. Ghidini, A., Sirtori, M., Romero, R., Hobbins, J., Prenatal diagnosis of pentalogy of Cantrell.J Ultrasound Med 1988; 7:567-572.

\section{AGRADECIMIENTOS}

A la doctora Elvia Goez, patóloga U de A. A la doctora Marta Lucía Echeverri L., ecocardiografista infantil del HUSVP. Por su valiosa ayuda en el manejo de estas pacientes y en la realización de este reporte. 